\title{
Superposition of semiconductor and semi-metal properties of self-assembled 2D $\mathrm{SnTiS}_{3}$ heterostructures
}

\author{
Srinivasa Reddy Tamalampudi ${ }^{1,2,3,7}$, Jin-You Lu $\mathbb{B}^{1,2,7 凶}$, Nitul Rajput $^{1,2}$, Chia-Yun Lai ${ }^{4}$, Boulos Alfakes ${ }^{1,2}$, Raman Sankar $\mathbb{D}^{5}$, \\ Harry Apostoleris ${ }^{1,2}$, Shashikant P. Patole $\mathbb{E}^{6}$, Ibraheem Almansouri ${ }^{1}$ and Matteo Chiesa $\mathbb{E}^{1,2,4 凶}$
}

Two-dimensional metal dichalcogenide/monochalcogenide thin flakes have attracted much attention owing to their remarkable electronic and electrochemical properties; however, chemical instability limits their applications. Chemical vapor transport (CVT)synthesized $\mathrm{SnTiS}_{3}$ thin flakes exhibit misfit heterojunction structure and are highly stable in ambient conditions, offering a great opportunity to exploit the properties of two distinct constituent materials: semiconductor $\mathrm{SnS}$ and semi-metal $\mathrm{TiS}_{2}$. We demonstrated that in addition to a metal-like electrical conductivity of $921 \mathrm{~S} / \mathrm{cm}$, the $\mathrm{SnTiS}_{3}$ thin flakes exhibit a strong bandgap emission at $1.9 \mathrm{eV}$, owing to the weak van der Waals interaction within the misfit-layer stackings. Our work shows that the misfit heterojunction structure preserves the electronic properties and lattice vibrations of the individual constituent monolayers and thus holds the promise to bridge the bandgap and carrier mobility discrepancy between graphene and recently established 2D transition metal dichalcogenide materials. Moreover, we also present a way to identify the top layer of $\mathrm{SnTiS}_{3}$ misfit compound layers and their related work function, which is essential for deployment of van der Waals misfit layers in future optoelectronic devices.

npj 2D Materials and Applications (2020)4:23 ; https://doi.org/10.1038/s41699-020-0158-7

\section{INTRODUCTION}

The library of atomically thin two-dimensional (2D) materials is continually expanding with the discovery of new naturally existing 2D materials beyond graphene. Of specific interest are those that combine high carrier mobility with a large bandgap ${ }^{1}$. Many studies have explored the unique properties of 2D material structures composed of transition metal chalcogenides and transition metal dichalcogenides and demonstrated their applications in a wide range of fields ${ }^{2}$. Among these $e^{3,4}$, single and few-layer $\mathrm{TiS}_{2}$ have been demonstrated as potential electrode candidates for lithium-sodium batteries ${ }^{5}$, pseudo-capacitors ${ }^{6}$, and solar cells owing to their high electrical conductivity ${ }^{7}$. Studies on SnS thin flakes have revealed many exotic effects, including a thicknesstunable optical bandgap of 1.0-1.6 eV and anisotropic electronic properties ${ }^{4}$. However, the fabrication of chemically stable monolayers of $\mathrm{SnS}$ or $\mathrm{TiS}_{2}$ has presented major challenges ${ }^{9,10}$. For instance, SnS exhibits high chemical reactivity due to the unshared electron pair in the group-IV atoms, which rapidly leads to surface oxidation ${ }^{9} . \mathrm{TiS}_{2}$ thin flakes also degrade rapidly in ambient or water and spontaneously convert to various titanium oxides by releasing $\mathrm{H}_{2} \mathrm{~S}^{10}$. A possible strategy to overcome this challenge is through encapsulation, which has yielded stable van der Waals heterostructures in ambient conditions ${ }^{11}$. Nevertheless, these human-made van der Waals heterostructures exhibit some limitations due to interface residues and misalignment of crystal orientation $^{12}$.

Misfit-layer compounds are an alternative to artificial stacking of van der Waals heterostructures ${ }^{13,14}$. As-deposited $\mathrm{SnTiS}_{3}$, for example, grows by chemical transport reaction through the formation of a spontaneous alternating monolayer stack of the constituent $2 \mathrm{D}$ materials $\mathrm{SnS}$ and $\mathrm{TiS}_{2}$. These alternating stacked layers exhibit greater chemical stability than either of its component layers, separately ${ }^{15}$. This type of self-assembled heterostructure is usually referred to as a misfit-layer compound ${ }^{16}$, and it is feasible to increase the thickness of one or both of the constituent layers in the repeated units in ferecrystals ${ }^{17}$. Both misfit and ferecrystals possess, in its bulk form, excellent properties for thermoelectric ${ }^{18}$, superconductor ${ }^{19}$, and intercalation applications ${ }^{20}$. However, despite the recent interest in misfitlayer compounds, very few studies explore their optical/electrical properties at low dimensions, particularly $\mathrm{SnTiS}_{3}$, which is a unique layered material combining semi-metal and semiconductor components.

This study was thus undertaken to provide a comprehensive understanding of the electronic band structure, optical properties, and crystal structure of $\mathrm{SnTiS}_{3}$ using electrical, ultraviolet/visible (UV/VIS), photoluminescence (PL), and Raman spectroscopy characterization. The measured results are interpreted with the help of first-principles density functional theory calculations. We found that electronic band structure and lattice vibrations of $\mathrm{SnTiS}_{3}$ are nearly a superposition of $\mathrm{SnS}$ and $\mathrm{TiS}_{2}$. As compared to the strong chemical bonding within component layers, the weak van der Waals bonding between the interlayers causes the $\mathrm{SnTiS}_{3}$ misfit system to simultaneously preserve the semiconductor and semi-metal properties of $\mathrm{SnS}$ and $\mathrm{TiS}_{2}$, which potentially leads to a new 2D materials with high mobility $\left(26.7 \mathrm{~cm}^{2} / \mathrm{Vs}\right)$ and a large bandgap $(\sim 1.9 \mathrm{eV})$. Our characterization results of $\mathrm{SnTiS}_{3}$ thin flakes show that in addition to measured high electrical

\footnotetext{
${ }^{1}$ Laboratory for Energy and NanoScience (LENS), Khalifa University of Science and Technology, Masdar Campus, PO Box 54224 Abu Dhabi, United Arab Emirates. ${ }^{2}$ Department of Mechanical and Materials Engineering, Khalifa University of Science and Technology, Masdar Campus, PO Box 54224 Abu Dhabi, United Arab Emirates. ${ }^{3}$ Department of Electrical and Computer Engineering, New York University Abu Dhabi, P.O. Box 129188, Abu Dhabi, United Arab Emirates. ${ }^{4}$ Department of Physics and Technology, UiT The Arctic University of Norway, 9010 Tromsø, Norway. ${ }^{5}$ Institute of Physics, Academia Sinica, Taipei 11529, Taiwan ROC. ${ }^{6}$ Department of Physics, Khalifa University of Science and Technology, Main Campus, PO Box 127788 Abu Dhabi, United Arab Emirates. ${ }^{7}$ These authors contributed equally: Srinivasa Reddy Tamalampudi, Jin-You Lu. ${ }^{\circledR}$ email: chun.lu@ku. ac.ae; matteo.chiesa@ku.ac.ae
} 
a

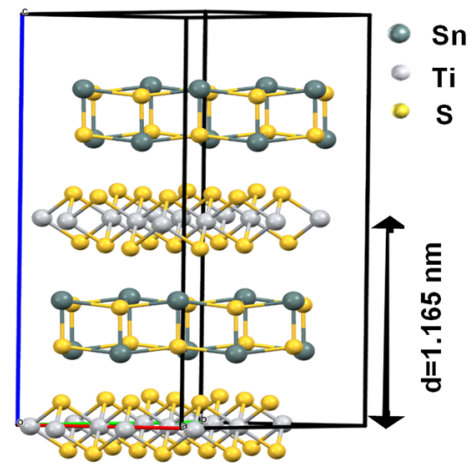

b

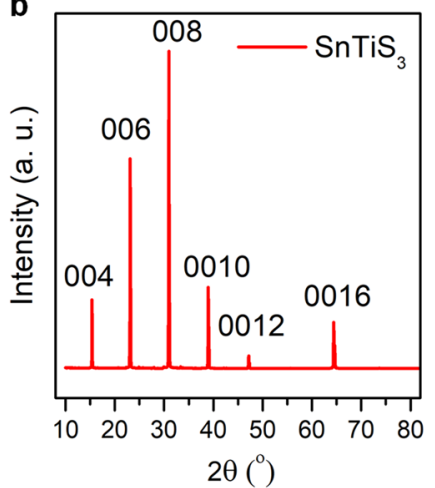

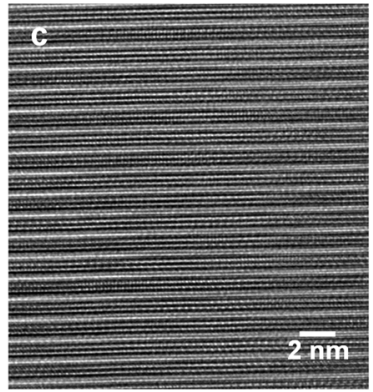
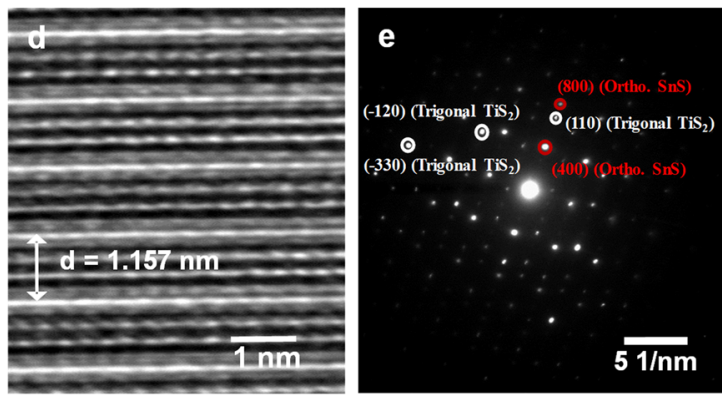

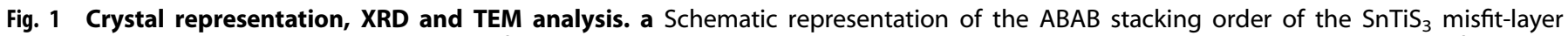
compound. b Single-crystal XRD spectrum of the grown $\mathrm{SnTiS}_{3}$ crystals. c Cross-sectional HRTEM image indicating the presence of periodic layers. d A magnified view of the alternating layers of $\mathrm{SnS}$ and $\mathrm{TiS}_{2}$. e A diffraction pattern collected from the zone axis [002].

conductivity, the photoluminescence spectra of $\mathrm{SnTiS}_{3}$ thin flakes reveal a bandgap emission, which is only ambiguously observed in bulk $\mathrm{SnS}^{21}$. Furthermore, we propose and demonstrate a way to identify the exposed component layer of the exfoliated $\mathrm{SnTiS}_{3}$ flake, utilizing atomic force microscopy (AFM) and Kelvin probe force microscopy (KPFM), for the reliable design and manufacture of optoelectronic devices.

\section{RESULTS AND DISCUSSIONS}

\section{Crystal characterization}

$\mathrm{SnTiS}_{3}$ grown by chemical vapor transport (CVT) naturally assumes a heterostructure of alternating $\mathrm{SnS}$ and $\mathrm{TiS}_{2}$ layers ${ }^{22}$. Specifically, the triclinic structure is composed of two kinds of mutually stacked layers: two-atom-thick orthorhombic SnS layers with distorted $\mathrm{NaCl}$ type and three-atom-thick trigonal $\mathrm{TiS}_{2}$ subsystem ${ }^{23}$. We begin our study with a bulk crystal of $\mathrm{SnTiS}_{3}$, whose crystal structure is shown in Fig. 1a. We confirm its structure and composition via single-crystal X-ray diffraction (XRD), powder XRD, and energy-dispersive $X$-ray spectroscopy (EDS), as reported in Fig. $1 \mathrm{~b}$, Supplementary Figs. 1 and 2, respectively. The EDSdetermined stoichiometric ratio of Sn:Ti:S compounds depends on the measured area, and the ratios at various locations on the grown crystal are $\sim 1: 1: 3$. The comparison of the measured XRD data of $\mathrm{SnTiS}_{3}$ crystal with the reported XRD data of $\mathrm{SnS}^{24}$ and $\mathrm{TiS}_{2}{ }^{25}$ confirms the existence of the misfit phase in the grown crystal. The measured powder XRD spectrum of $\mathrm{SnTiS}_{3}$ in Supplementary Fig. 1 indicates that $a=b=9.017$, and $c=23.29 \AA$ with $a=88.11^{\circ}$ and $\gamma=37.75^{\circ}$ in a triclinic crystal structure, which is in accordance with the trigonal phase of the $\mathrm{TiS}_{2}$ subsystem having $a=3.417, b=3.417$, and $c=5.823 \AA$ and with an orthorhombic phase of SnS having $a=4.024, b=4.443$, and $c=11.68 \AA$, respectively. Since the unit cell parameters for the $\mathrm{SnS}$ and $\mathrm{TiS}_{2}$ are mutually incommensurate, the layer misfit must cause a certain strain effect on one of the subsystems relative to the other ${ }^{19}$. The misfit angle between the $\mathrm{SnS}$ and $\mathrm{TiS}_{2}$ layers as calculated from the structure file is $1.88^{\circ}$.
Figure 1c shows the transmission electron microscopy (TEM) image collected from a lamella. The cross-sectional image suggests the presence of periodically alternating layers. The magnified view from the high-resolution (HR) TEM image of the sample in Fig. 1d exhibits the atomic structure of the layers and the periodic alternation between the $\mathrm{SnS}$ and $\mathrm{TiS}_{2}$ layers. The heterostructure shows a periodicity of $2.314 \mathrm{~nm}$ along the $c$-axis, which agrees with our XRD characterization results. A diffraction pattern collected from [002] zone axis is shown in Fig. 1e, which matches with the misfit-layer compound studied elsewhere ${ }^{26}$. Specific diffraction spots originating from the trigonal phase of $\mathrm{TiS}_{2}$ and the orthorhombic phase of SnS are indicated in Fig. 1e. The HRTEM results are in agreement with the image simulated using JEMS software by inputting the structure information retrieved from the XRD measurement, as displayed in Supplementary Fig. 3. The plane view of the HRTEM image of the singlecrystal $\mathrm{SnTiS}_{3}$ in Supplementary Fig. 4a shows a large area of periodic and uniform structures of $\mathrm{SnTiS}_{3}$. The magnified view of the aforementioned HRTEM image is shown in Supplementary Fig. $4 \mathrm{~b}$. Furthermore, the core-loss spectrum in electron energy loss spectroscopy measurements, as obtained from the grown crystal, confirms the presence of the elements present in the material (Supplementary Fig. 5).

\section{DFT simulations}

We first theoretically investigate the monolayer and bulk band structure of individual $\mathrm{SnS}, \mathrm{TiS}_{2}$, and $\mathrm{SnTiS}_{3}$ layers. The GGA Perdew-Burke-Ernzerhof exchange-correlation functional is used for all density functional theory (DFT) electronic calculations. We obtain an indirect bandgap of $0.64 \mathrm{eV}$ for bulk SnS, which increases to $1.38 \mathrm{eV}$ for monolayer SnS, as shown the Fig. 2 a. For the $\mathrm{TiS}_{2}$ layer, both bulk and monolayer are semi-metals with a small overlap between the conduction and valence bands, as shown in Fig. 2b. Figure 2c shows that the calculated electronic band structures of the monolayer of $\mathrm{SnTiS}_{3}$ misfit are nearly a superposition of $\mathrm{SnS}$ and $\mathrm{TiS}_{2}$ in the misfit triclinic crystal structure (Supplementary Fig. 6a, b), which exhibits an effective semi- 

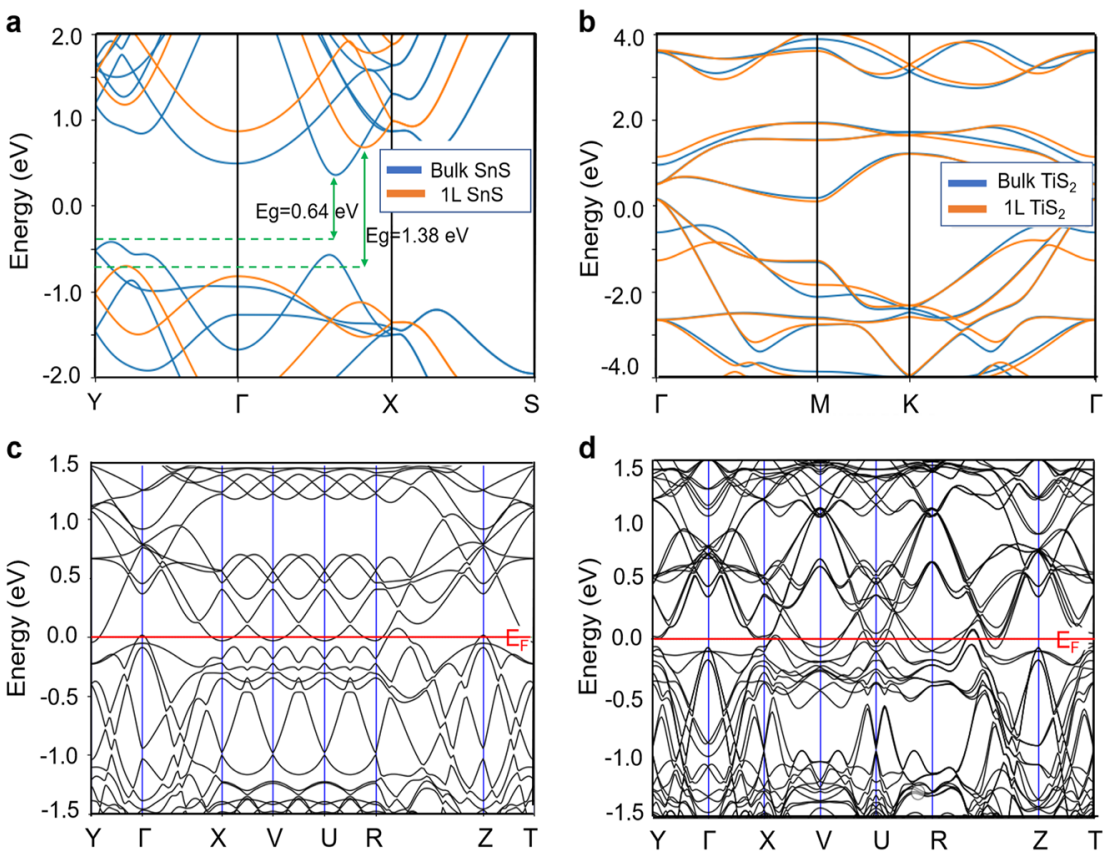

Fig. 2 DFT simulations of bulk and monolayer individual SnS, $\mathrm{TiS}_{2}$, and $\mathrm{SnTiS}_{3}$. a Electronic band structures of bulk and monolayer SnS in the orthorhombic crystals. b Electronic band structures of bulk and monolayer $\mathrm{TiS}_{2}$ in the trigonal crystals. DFT-predicted electronic band structure of $\mathbf{c}$ monolayer and $\mathbf{d}$ bulk thickness of $\mathrm{SnTiS}_{3}$.

a

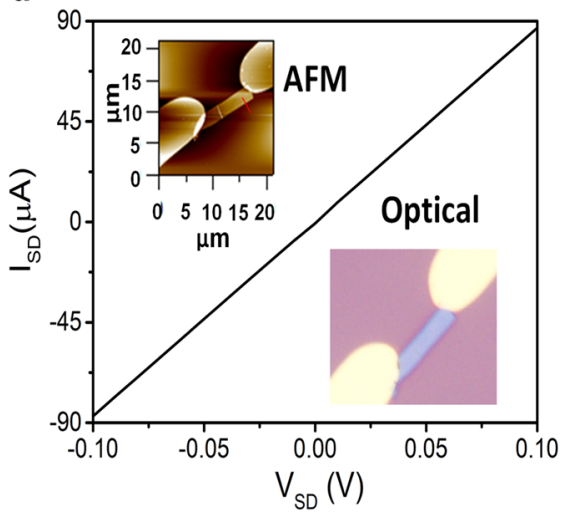

b

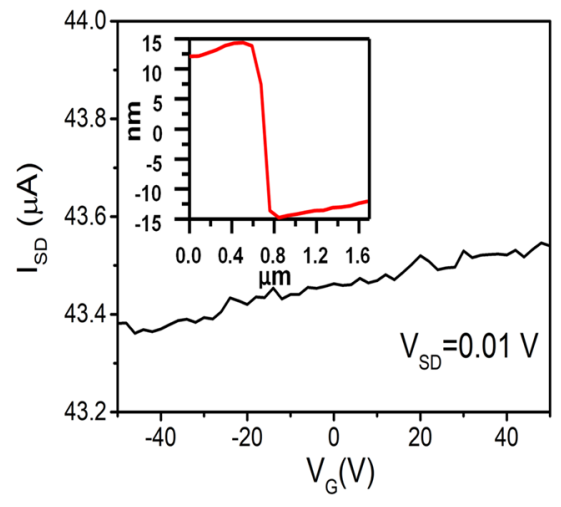

Fig. 3 Electrical characterization at room temperature $(T=300 \mathrm{~K})$ of an $\mathrm{SnTiS}_{3}$ FET. a The measured output electrical characteristics of misfit $\mathrm{SnTiS}_{3}$ layers. The optical and AFM profile image of the fabricated devices shows in the inset. b Transfer characteristics of SnTiS 3 FET at $V_{\mathrm{sd}}=0.01 \mathrm{~V}$. The fabricated device thickness is $30 \mathrm{~nm}$ as shown in the inset of the inset.

metallic property owing to the contribution of the $\mathrm{TiS}_{2}$. The overlapped area of bulk $\mathrm{SnTiS}_{3}$ layers in Fig. $2 \mathrm{~d}$ is increased, as compared to that of monolayer $\mathrm{SnTiS}_{3}$, and thus exhibits more conductive properties. We have also observed a similar trend for the layer-dependent electronic band structure of $\mathrm{SnTiS}_{3}$ predicted by the DFT simulation, as shown in Supplementary Fig. 7.

Device fabrication and electrical characterization

To evaluate the electrical characteristics of the material, we have fabricated field-effect transistors (FETs) of $\mathrm{SnTiS}_{3}$ thin flakes using mechanical exfoliation. The output characteristic $\left(V_{s d} v s I_{s d}\right)$ of an $\mathrm{SnTiS}_{3}$ FET with a thickness of $30 \mathrm{~nm}$ shows that the electrical conductivity of thin $\mathrm{SnTiS}_{3}$ flakes reaches $921 \mathrm{~S} / \mathrm{cm}$, which is comparable to that of metal-like 2D MXene ${ }^{27}$. The measured $V_{\text {sd }}$ Vs $I_{\text {sd }}$ curves also exhibits that $\mathrm{SnTiS}_{3}$ thin flake forms an ohmic contact with the deposited $\mathrm{Cr} / \mathrm{Au}(5 / 40 \mathrm{~nm})$ metal electrodes, irrespective of the exposed top surface. The optical image and AFM characterized height profile of the fabricated devices are shown in the insets of Fig. 3a, b, respectively. The measured back gate voltage-dependent transfer characteristic of the $\mathrm{SnTiS}_{3}$ FET does not change with applying a gate voltage from -50 to $+50 \mathrm{~V}$ at a fixed voltage bias $\left(V_{s d}\right)$ of $0.01 \mathrm{~V}$, which indicates abundant charge carrier in the channel. It cannot be switched off within the applied experimental gate voltage range, as shown in Fig. 3b.

Optical characterization

Following the results of electrical characterization, we study the optical properties of misfit $\mathrm{SnTiS}_{3}$ by preparing a suspension of flakes in isopropyl alcohol and then measuring the sample via UVVIS transmittance spectrometry. In Fig. 4a, the absorbance spectrum of the flake-IPA mixture shows an absorption peak near $\sim 620 \mathrm{~nm}$, indicating the presence of a direct bandgap with the energy of around 1.9-2.3 eV after fitting with a Tauc's plot of $(a h v)^{2} \propto h v$, as shown in the inset of the Fig. 4a. For an indirect bandgap, the Tauc plot has the relation of $(a h v)^{1 / 2} \propto h v$, as shown in the study of SnS nanosheets ${ }^{8}$. Moreover, the fluorescence 
a

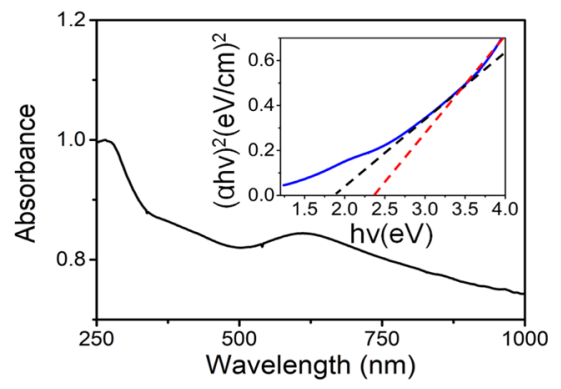

C

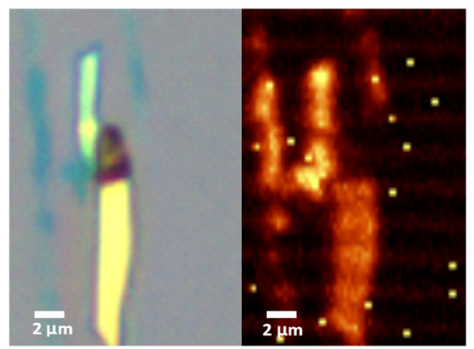

e

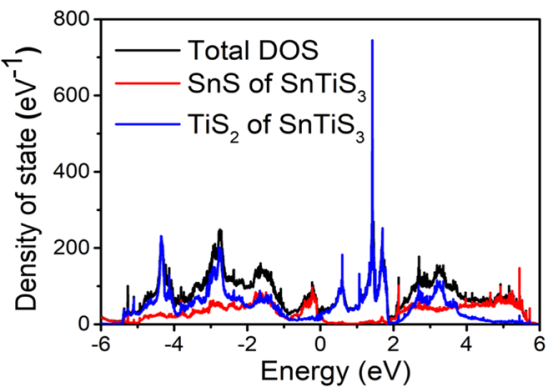

b

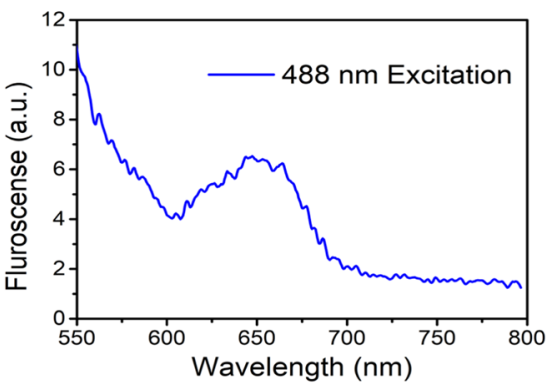

d

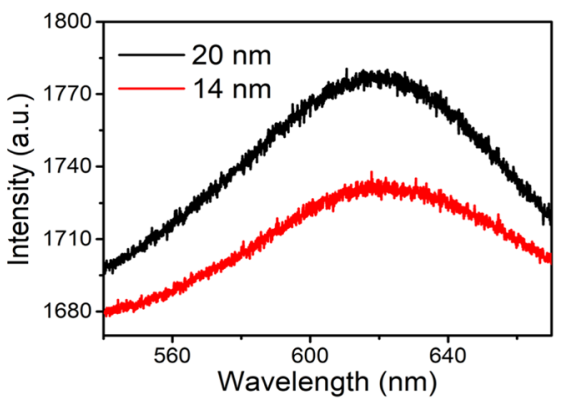

f

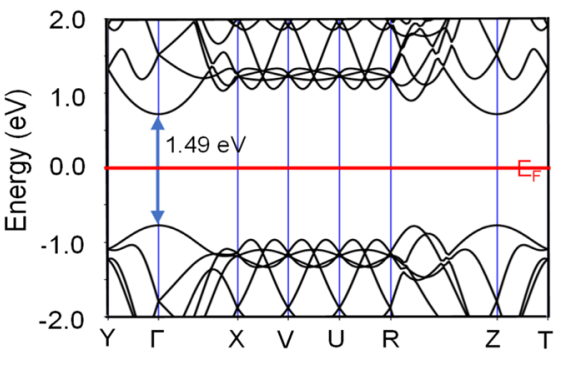

Fig. 4 Optical characterization of the $\mathrm{SnTiS}_{3}$ crystals. a Absorbance spectrum of the exfoliated $\mathrm{SnTiS}_{3}$ flakes in iso-propanol solvent. The bandgap of the $\mathrm{SnTiS}_{3}$ crystal is estimated to be 1.9-2.3 eV by fitting the measured data to a Tauc's plot as shown in inset. $\mathbf{b}$ Fluorescence spectrum of the $\mathrm{SnTiS}_{3}$ crystal flakes by exciting with $488 \mathrm{~nm}$ laser source. c Optical image of the mechanically exfoliated few-layer thickness SnTiS3 flakes (left-hand side), with the corresponding photoluminescence mappings (right-hand side). d Photoluminescence spectrum of $\mathrm{SnTiS}_{3}$ crystal with different layer thicknesses. e Calculated density of state of five-layer $\mathrm{SnTiS}_{3}$ and partial density of state contributed by its component $\mathrm{SnS}$ and $\mathrm{TiS}_{2}$ layers. f Calculated electronic band structures of monolayer $\mathrm{SnS}$ in the misfit triclinic $\mathrm{SnTiS}_{3} \mathrm{crystal}$ structure, which show a direct energy gap.

spectrum of the suspension under $488 \mathrm{~nm}$ laser excitation was measured. The fluorescence peak in the visible range in Fig. $4 \mathrm{~b}$ represents a bandgap emission of $\sim 1.9 \mathrm{eV}$, which matches the position of the retrieved optical bandgap of $\sim 1.9 \mathrm{eV}$ from the absorption spectrum.

We perform photoluminescence measurements over exfoliated $\mathrm{SnTiS}_{3}$ flakes on $\mathrm{Si} / \mathrm{SiO}_{2}$ substrate. Notably, few-layer $\mathrm{SnTiS}_{3}$ is obtained as needle-like flakes with a large length-to-width aspect ratio, similar to the reported franckeite misfit-layer exfoliation ${ }^{28}$. The optical microscope images of several flakes with different thicknesses in Fig. 4c are shown alongside their corresponding PL map, shown in Fig. 4d. The thicknesses of the flakes are 10 and 14 layers (14- and 20-nm-thick surfaces). The PL spectra of exfoliated $\mathrm{SnTiS}_{3}$ flakes show a strong PL peak at $\sim 620 \mathrm{~nm}(\sim 2 \mathrm{eV})$, which indicates that the interlayer carrier transport between component $\mathrm{SnS}$ and $\mathrm{TiS}_{2}$ layers is not efficient enough to avoid the recombination of photogenerated electron-hole pairs in SnS layers of $\mathrm{SnTiS}_{3}$.

To explore why electronically metallic $\mathrm{SnTiS}_{3}$ thin flakes have a bandgap emission, we analyze the partial density of states contributed by their component layers, as shown in Fig. 4e. Our
DFT simulation shows that the bandgap emission results from the SnS component layer, which possesses a bandgap at near $1.4 \mathrm{eV}$. It is interesting to compare the electronic band structure of monolayer SnS in its intrinsic orthorhombic and misfit triclinic crystal structures since it has been reported that the orthorhombic SnS does not exhibit bandgap emission due to its indirect bandgap properties ${ }^{29}$. Our DFT simulation shows that in the misfit crystal structure of $\mathrm{SnTiS}_{3}$, SnS contributes a direct bandgap with the energy of $1.49 \mathrm{eV}$, as shown in Fig. 4f. For comparison, we have included the band structure of SnS layers in its intrinsic orthorhombic crystal phase as a function of layer number in Supplementary Fig. $6 c$, d, showing that its value of an indirect bandgap increases from 0.64 to $1.39 \mathrm{eV}$ with decreasing the layer number from bulk to monolayer. It should be noted that the absolute value of the DFT-predicted bandgap is underestimated in typical GGA-DFT simulations, but the relative trend is correct $^{30}$. Owing to the weak van der Waals bonding among misfit layers, here we have observed, naturally grown van der Waals $\mathrm{SnTiS}_{3}$ heterostructures exhibiting photoluminescent emissions. The electronic and optical properties of $\mathrm{SnS}_{1} \mathrm{TiS}_{2}$, and $\mathrm{SnTiS}_{3}$ thin flakes are summarized in Table 1, respectively. 
Table 1. Comparison of electronic and optical properties of $\mathrm{SnS}, \mathrm{TiS}_{2}$, and $\mathrm{SnTiS}_{3}$.

\begin{tabular}{llll}
\hline Material & SnS & $\mathrm{TiS}_{2}$ & $\mathrm{SnTiS}_{3}$ \\
\hline Electronic & Indirect bandgap & Semi-metal & Semi-metal \\
Optical & Bandgap in the range of 1.0-1.6 eV & Bandgap in the range of $0.3-0.5 \mathrm{eV}^{32}$ & Bandgap emission at 1.9 eV (this work) \\
\hline
\end{tabular}

a

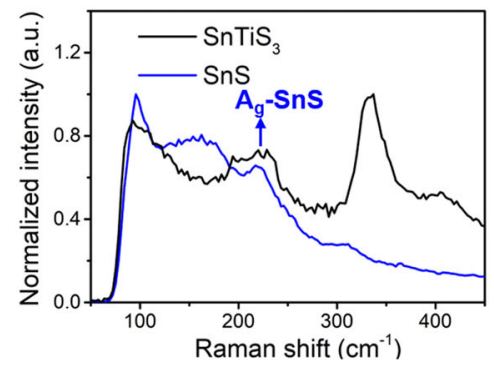

C

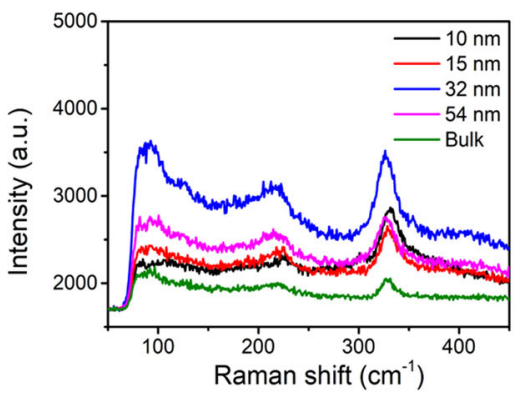

b

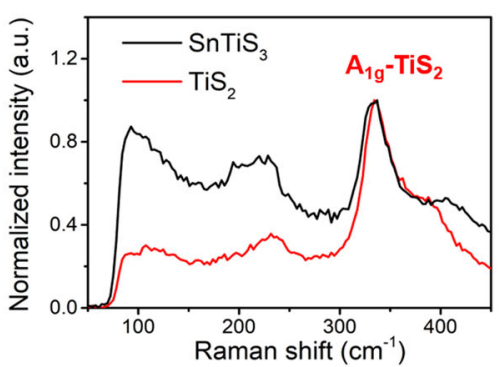

d

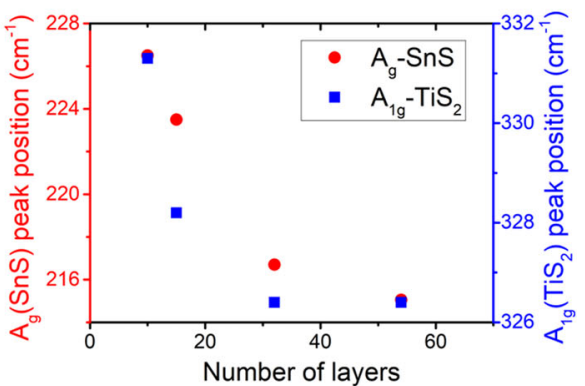

Fig. 5 Raman spectroscopy characterization of the bulk and few-layer $\mathrm{SnTiS}_{\mathbf{3}}$ flakes. a, $\mathbf{b}$ Phonon modes of the $\mathrm{SnS}_{1} \mathrm{TiS}_{2}$, and as-grown bulk $\mathrm{SnTiS}_{3}$ crystal. c Raman spectra of $\mathrm{SnTiS}_{3}$ with different layer thickness under excitation of $532 \mathrm{~nm}$ laser light. d $\mathrm{A}_{\mathrm{g}}$ and $\mathrm{A}_{1 \mathrm{~g}}$ Raman peak positions of $\mathrm{SnTiS}_{3}$ with different number of layers.

\section{Raman spectroscopy characterization}

Furthermore, we have performed Raman spectroscopy measurements on $\mathrm{SnTiS}_{3}$ flakes. Figure $5 \mathrm{a}, \mathrm{b}$ shows the Raman-active vibrational modes of bulk SnS, $\mathrm{TiS}_{2}$, and $\mathrm{SnTiS}_{3}$, respectively. We observe that the Raman modes of bulk $\mathrm{SnTiS}_{3}$ are also nearly a superposition of bulk $\mathrm{SnS}$ and $\mathrm{TiS}_{2}$. The bulk SnS exhibits $\mathrm{B}_{3 g}, \mathrm{~A}_{\mathrm{g}}$ and $A_{g}$ Raman modes at 153,220 , and $294 \mathrm{~cm}^{-1}$, which agree with the DFT simulation in Supplementary Fig. 8a. Similarly, the Raman spectrum of Bulk-TiS shows two Raman active modes consisting of an in-plane vibrational mode $\left(E_{g}\right)$ at $233 \mathrm{~cm}^{-1}$ and an out-ofplane mode $\left(A_{1 g}\right)$ at $328 \mathrm{~cm}^{-1}$, which are also in line with DFTprediction in Supplementary Fig. 8b. As it comes to $\mathrm{SnTiS}_{3}$ thin flakes, the measured Raman spectrum of $\mathrm{SnTiS}_{3}$ is dominated by SnS vibrations with corresponding peaks at 94 and $222 \mathrm{~cm}^{-1}$ $\left(A_{\mathrm{g}-\mathrm{SnS}}\right)$ as well as $\mathrm{TiS}_{2}$ vibration at $330 \mathrm{~cm}^{-1}\left(\mathrm{~A}_{1 \mathrm{~g}-\mathrm{TiS} 2}\right)$. It should be noted that the $\mathrm{SnS}$ and $\mathrm{TiS}_{2}$ thin flakes are quickly degraded under the laser illumination. Several Raman active modes of $\mathrm{SnS}$ and $\mathrm{TiS}_{2}$ disappeared owing to structural modulation between $\mathrm{SnS}$ and $\mathrm{TiS}_{2}$ layers. Moreover, we can observe that as laser energy is larger than $1.9 \mathrm{eV}(620 \mathrm{~nm})$ in Supplementary Fig. $8 c$, the measured Raman spectra exhibit resonant Raman background features, which results from the bandgap emission from the component $\mathrm{SnS}$ layers. Thus, those peaks related to the SnS exhibit comparatively stronger intensities than that of $\mathrm{TiS}_{2}$ as the laser energy is increased, which results from the absorption of the bandgap of SnS component layers (1.9 eV bandgap).

The relationships between the intensities of Raman peaks and the thickness of the layered $\mathrm{SnTiS}_{3}$ crystal flakes have also been investigated. The intensity ratio of $A_{1 g-T i s 2} / A_{g-S n s}$ for bulk $\mathrm{SnTiS}_{3}$ is close to 1, which gradually increases to 2.5 for thin layers, as shown in Fig. 5c. This can be attributed to the reduction or enhancement of scattering centers for out-of-plane crystal scattering, as the thickness decreases down to the nanoscale. It can also be explained by an optical interference in the 2D crystal/ $\mathrm{SiO}_{2} / \mathrm{Si}$ system and corresponding optical field enhancement for certain 2D crystal thicknesses. Significant changes in the frequency of these Raman modes are observed for different $\mathrm{SnTiS}_{3}$ thicknesses, as shown in Fig. $5 \mathrm{~d}$.

AFM and KPFM studies to identify the exposed top surface Unlike the exfoliation of a homogeneous 2D material structure, in $\mathrm{SnTiS}_{3}$ the exposed top surface could be either SnS or TiS 2 . Due to the high transparency of $2 \mathrm{D}$ flakes, identifying the top exposed surface of misfit-layer compounds with the help of optical microscopy and Raman spectroscopy is difficult and assumed as a cumbersome approach ${ }^{31}$. Here, we propose a way to infer the nature of the exposed material using nanoscopic AFM characterization since the AFM-based force of adhesion and KPFM-derived work function offer a greater opportunity to explore the terminating layer properties ${ }^{32,33}$. The AFM-derived force of adhesion $F_{\mathrm{AD}}$ or surface energy $\gamma\left(F_{\mathrm{AD}}=4 \pi R \gamma\right.$, where $R$ is the tip radius) statistically show two distinct distributions indicating either SnS or $\mathrm{TiS}_{2}$ are exposed to the ambient, as shown in Supplementary Fig. 9. Comparing the mean force of adhesion at two distributions and the DFT-predicted adsorption energy of water molecules on $\mathrm{SnS}$ and $\mathrm{TiS}_{2}$-terminated $\mathrm{SnTiS}_{3}$ surface in Fig. $6 \mathrm{~b}$ (further information in Supporting Information), we can infer that the high surface energy distribution results from 
a

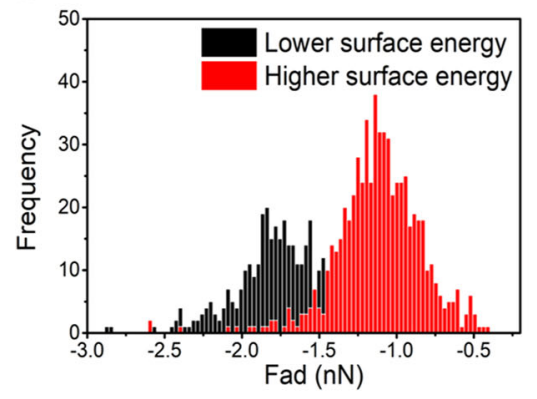

b

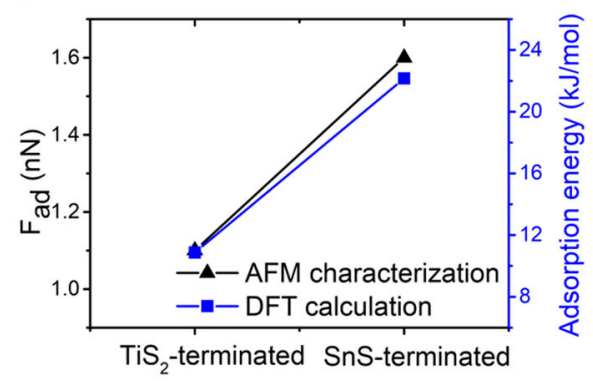

C

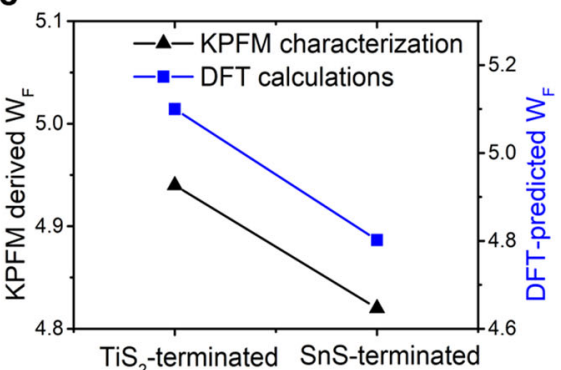

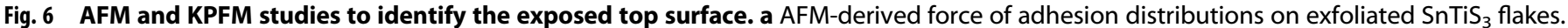
b DFT-predicted adsorption energy of water molecules on $\mathrm{SnS}$ and $\mathrm{TiS}_{2}$-terminated $\mathrm{SnTiS}_{3}$ surfaces. c KPFM-derived work function on exfoliated $\mathrm{SnTiS}_{3}$ surface and DFT-predicted work function of $\mathrm{SnS}$ and $\mathrm{TiS}_{2}$-terminated $\mathrm{SnTiS}_{3}$ surfaces.

scanning the SnS on the top, while the low surface energy corresponds to the $\mathrm{TiS}_{2}$-terminated stack surface.

We furthermore identified the work function of exfoliated $\mathrm{SnTiS}_{3}$ using KPFM scanning, as shown in Supplementary Fig. 10. The measured work functions show two different values 4.82 and $4.94 \mathrm{eV}$ for exfoliated $\mathrm{SnTiS}_{3}$ layers. The height maps corresponding to the KPFM scanning regions are included in Supplementary Fig. 11. In comparison with the DFT-predicted work functions (Supplementary Fig. 12) and measured work function, we can infer that $\mathrm{TiS}_{2}$-terminated $\mathrm{SnTiS}_{3}$ surface possesses a higher work function than that of SnS-terminated $\mathrm{SnTiS}_{3}$ surface, as shown in the Fig. 6c. Deducing the nature of the exposed surface is mandatory for the robust and repeatable fabrication of ohmic contact type devices since the work function of the two surfaces is different. This identification method of the combination of AFM and KPFM is critical for future atomically thin van der Waals heterostructures in the electronics and optoelectronics applications. Further experimental details are available in Supporting Information.

\section{DISCUSSION}

We have performed electrical, optical, $\mathrm{PL}$, and Raman spectroscopy studies on mechanically exfoliated $\mathrm{SnTiS}_{3}$ thin flakes. The exfoliated $\mathrm{SnTiS}_{3}$ thin flakes show a high electrical conductivity resulting from the $\mathrm{TiS}_{2}$ component layers, while also exhibiting visible PL emission from the SnS component layer of $\mathrm{SnTiS}_{3}$ crystal, indicating the presence of a direct bandgap of $1.9 \mathrm{eV}$. These results suggest the presence of both conducting and semiconducting properties in the material simultaneously. In combination with first-principles calculations, we show that the interlayer van der Waals interaction preserves the properties of semi-metal $\mathrm{TiS}_{2}$ and a direct bandgap semiconductor $\mathrm{SnS}$. In this way, SnTiS3 represents a potential realization of the promise of 2D materials to combine high carrier mobility and a large bandgap in a single material, with wide-ranging application possibilities. With an eye towards such applications, we have combined AFM and KPFM scanning on $\mathrm{SnTiS}_{3}$ thin flakes with DFT simulation to demonstrate a way to determine the top layer composition of the
$\mathrm{SnTiS}_{3}$ stack and obtain the related work functions. This characterization represents an important step toward exploring the intriguing properties of $\mathrm{SnTiS}_{3}$ and its potential applications in future optoelectronic devices.

\section{METHODS}

Crystal growth

CVT method employed $\mathrm{TeCl}_{4}$ as the source of transport agent $\left(\mathrm{Cl}_{2}\right)$, which allows an effective and faster vapor transport to achieve the necessary supersaturation of the expected final product. A three-zone muffle furnace was used for this purpose, having typical temperature profiles for the growth of single crystals. A stoichiometric amount of Sn:Ti:S $=1: 1: 3.1(5 \mathrm{~N}$ purity for $\mathrm{Sn}, \mathrm{Ti}$, and $\mathrm{S}$ ) was sealed into an evacuated quartz ampoule and heated for 4 days at $650^{\circ} \mathrm{C}$. About $10 \mathrm{~g}$ of the pre-reacted $\mathrm{SnTiS}_{3}$ powder was placed together with a variable amount of $\mathrm{TeCl}_{4}$ (purity $\left.4 \mathrm{~N}\right)(100 \mathrm{mg}$ ) at one end of the silica ampoule (length $35 \mathrm{~cm}$ with an inner diameter of $2.00 \mathrm{~cm}$ and an outer diameter of $2.2 \mathrm{~cm}$ ). The chlorine concentration in the range of $2.6-5.7 \mathrm{mg}$ was yielded sufficiently from the $\sim 100 \mathrm{mg} \mathrm{TeCl} /$ $\mathrm{cm}^{3}$, which provided high transport rates of near $150 \mathrm{mg}$ per day. All preparation steps before the quartz tube evacuated flame sealing were carried out in an Argon gas-filled glove box of oxygen and water level kept below $\sim 1 \mathrm{ppm}$. The loaded ampoule was evacuated and flame-sealed before loading into the tube furnace for CVT growth. For the growth of $\mathrm{SnTiS}_{3}$ single crystals, the end of the ampoule containing the pre-reacted material was held at $900^{\circ} \mathrm{C}$, and the growth end was maintained at a temperature near $800^{\circ} \mathrm{C}$ with a temperature gradient near $2.5^{\circ} \mathrm{C} / \mathrm{cm}$ for 2 weeks. $\mathrm{SnTiS}_{3}$ single crystals of sizes up to $0.3 \times 0.3 \times 0.27 \mathrm{~mm}^{3}$ were obtained.

\section{UV-VIS spectroscopy}

The grown SnTiS3 crystal flakes were suspended in the IPA solvent using sonication before spectroscopy studies were performed using a Duetta Fluorescence and Absorbance Spectrometer. Scans were performed between 1100 and $250 \mathrm{~nm}$ with a spectral resolution of $1 \mathrm{~nm}$.

$\mathrm{PL}$ and Raman measurements

Raman and PL spectrums of a few layer and bulk $\mathrm{SnTiS}_{3}$ flakes were measured using WITEC Raman 300RS system with three different laser excitation wavelengths, including 633,532 , and $488 \mathrm{~nm}$, respectively. Due 
to the small width of thin $\mathrm{SntiS}_{3}$ crystals, only well-isolated flakes were used for Raman spectroscopy measurements to avoid signal overlapping with other regions. The low laser power density was used to avoid $\mathrm{SnTiS}_{3}$ degradation.

\section{AFM characterization}

The $\mathrm{SnTiS}_{3}$ flakes are measured with a Cypher AFM from Asylum Research in amplitude modulation mode. The standard OLYMPUS cantilevers (AC160TS) with $k \approx 30 \mathrm{~N} / \mathrm{m}, Q \approx 400$, and $f 0 \approx 250 \mathrm{kHz}$ were used for the force reconstruction. Sader-Jarvis-Katan formalism ${ }^{32-36}$ were employed, and the amplitude $A$ and phase lag $\Phi$ were recorded as a function of tipsample separation distance $d$. Free cantilever oscillation amplitude around $50 \mathrm{~nm}$ was used to avoid bistability with the trigger point around $47 \mathrm{~nm}$. Besides, as the tip radius $R$ is known to affect the tip-sample interaction force, it has been monitored continuously with the critical amplitude method $^{33}$ to ensure that there was no change in $R$ throughout the experiment. Five randomly picked flakes were examined, and for each of the flakes, around 300 measurements were collected.

\section{KPFM characterization}

KPFM mapping was performed in ambient using a Cypher AFM from Asylum Research. Conductive iridium coated AC tip $(k \approx 2.8 \mathrm{~N} / \mathrm{m}, f \approx 75 \mathrm{kHz})$ was utilized for this type of scans. No sample grounding was done before scanning. Similar to the acquisition of force, the initial drive frequency is determined with thermal analysis. When the probe is driven electrically close to the surface, an additional tune is performed which slightly changes the resonance frequency. The target amplitude and the trigger point were set to $1 \mathrm{~V}$ and $800 \mathrm{mV}$, respectively, as recommended by the manufacturer. The integral and proportional gain values were varied until a stable scan was obtained. The scan size varied based on the size of the measured flake, with slow scan rates in the range of $0.5-1 \mathrm{~Hz}$.

\section{TEM measurements}

Thin lamellae for TEM study were prepared using a standard in situ lift-out procedure ${ }^{37}$. A dual-beam FIB system (Thermo Fisher Scientific, Helios 650) was used to fabricate the lamellae. In order to protect the top surface layers from the ion beam damaging, the capping layer of $\mathrm{Pt}$ was deposited using the available precursor gas $\left.\left(\mathrm{CH}_{3}\right)_{3} \mathrm{Pt}\left(\mathrm{CpCH}_{3}\right)\right)$ in the dual-beam system. Two sets of lamellae were prepared to observe the plane view and cross-sectional view of the grown single-crystal $\mathrm{SnTiS}_{3}$. An image corrected TEM system (Thermo Fisher Scientific, Titan G2) operating at $300 \mathrm{kV}$ was used for the HRTEM imaging.

\section{Device fabrication}

Mechanically exfoliated $\mathrm{SnTiS}_{3}$ flakes were transferred to a cleaned $\mathrm{Si} / \mathrm{SiO}_{2}$ substrate and then spin-coated with photoresist (SPR220) and prebaked at $115^{\circ} \mathrm{C}$ for $90 \mathrm{~s}$. A direct laser lithography technique (Dilase 250) was used to write the metal pads on to the selected $\mathrm{SnTiS}_{3}$ flakes. The patterned sample was then post-baked at $120^{\circ} \mathrm{C}$ for $90 \mathrm{~s}$ and cooled down to ambient temperature before developing the pattern in the AZ725 developer for $40 \mathrm{~s}$. The sample was subsequently rinsed with DI water several times to remove any unwanted photoresist and developer residues. Next, the developed samples were transferred to the thermal evaporator (Lesker PVD 75 thermal evaporator) and $5 \mathrm{~nm}$ of $\mathrm{Cr}$ followed by $40 \mathrm{~nm}$ of Au were deposited at $1 \times 10^{-5}$ Torr. After the metal deposition, the lift-off process was carried out in acetone.

\section{Electrical characterization}

Room temperature electrical measurements of the as-fabricated $\mathrm{SnTiS}_{3}$ FETs were performed using a probe station (Signatone 1160 Manual Prober) equipped with a semiconductor parameter analyzer (Agilent B1505A curve tracer) at ambient conditions.

\section{DATA AVAILABILITY}

All of the relevant data are available from the authors. Requests for data and materials should be addressed to M.C.
Received: 11 February 2020; Accepted: 12 June 2020; Published online: 15 July 2020

\section{REFERENCES}

1. Bhimanapati, G. R. et al. Recent advances in two-dimensional materials beyond graphene. ACS Nano 9, 11509-11539 (2015).

2. Pop, E. et al. In 2017 IEEE 17th International Conference on Nanotechnology (IEEENANO), Pittsburgh, USA 916-917.

3. Ge, Y. et al. Broadband nonlinear photoresponse of 2D TiS2 for ultrashort pulse generation and all-optical thresholding devices. Adv. Opt. Mater. 6, 1701166 (2018).

4. Tian, Z., Guo, C., Zhao, M., Li, R. \& Xue, J. Two-dimensional SnS: a phosphorene analogue with strong in-plane electronic anisotropy. ACS Nano 11, 2219-2226 (2017)

5. Tao, $\mathrm{H}$. et al. TiS(2) as an advanced conversion electrode for sodium-ion batteries with ultra-high capacity and long-cycle life. Adv. Sci. 5, 1801021-1801021 (2018).

6. Muller, G. A., Cook, J. B., Kim, H.-S., Tolbert, S. H. \& Dunn, B. High performance pseudocapacitor based on 2D layered metal chalcogenide nanocrystals. Nano Lett. 15, 1911-1917 (2015).

7. Huckaba, A. J. et al. Low-cost TiS2 as hole-transport material for perovskite solar cells. Small Methods 1, 1700250 (2017).

8. Brent, J. R. et al. Tin(II) sulfide (SnS) nanosheets by liquid-phase exfoliation of herzenbergite: IV-VI main group two-dimensional atomic crystals. J. Am. Chem. Soc. 137, 12689-12696 (2015).

9. Higashitarumizu, N. et al. Self-passivated ultra-thin SnS layers via mechanical exfoliation and post-oxidation. Nanoscale 10, 22474-22483 (2018).

10. Vega-Mayoral, V. et al. Solvent exfoliation stabilizes TiS2 nanosheets against oxidation, facilitating lithium storage applications. Nanoscale 11, 6206-6216 (2019).

11. Tamalampudi, S. R. et al. Thickness-dependent resonant Raman and E' photoluminescence spectra of indium selenide and indium selenide/graphene heterostructures. J. Phys. Chem. C 123, 15345-15353 (2019).

12. Purdie, D. G. et al. Cleaning interfaces in layered materials heterostructures. Nat. Commun. 9, 5387 (2018).

13. Burzurí, E. et al. Simultaneous assembly of van der Waals heterostructures into multiple nanodevices. Nanoscale 10, 7966-7970 (2018).

14. Ryu, Y. K., Frisenda, R. \& Castellanos-Gomez, A. Superlattices based on van der Waals 2D materials. Chem. Commun. 55, 11498-11510 (2019).

15. Fang, C., de Groot, R. A., Wiegers, G. A. \& Haas, C. Electronic structure of the misfit layer compound (SnS)1.20TiS2: band structure calculations and photoelectron spectra. J. Phys. Condens. Matter 8, 1663-1667 (1996).

16. Lorenz, T., Joswig, J.-O. \& Seifert, G. Two-dimensional and tubular structures of misfit compounds: structural and electronic properties. Beilstein J. Nanotechnol. 5, 2171-2178 (2014).

17. Merrill, D. R., Moore, D. B., Bauers, S. R., Falmbigl, M. \& Johnson, D. C. Misfit layer compounds and ferecrystals: model systems for thermoelectric nanocomposites. Materials 8, 2000-2029 (2015).

18. Wan, C., Wang, Y., Wang, N. \& Koumoto, K. Low-thermal-conductivity $(\mathrm{MS})(1+\mathrm{x})$ $(\mathrm{TiS}(2))(2)(\mathrm{M}=\mathrm{Pb}, \mathrm{Bi}, \mathrm{Sn})$ misfit layer compounds for bulk thermoelectric materials. Materials 3, 2606-2617 (2010).

19. Sankar, R. et al. Superconductivity in a misfit layered (SnS)1.15(TaS2) compound. Chem. Mater. 30, 1373-1378 (2018).

20. Hernán, L., Lavela, P., Morales, J., Sánchez, L. \& Tirado, J. L. Intercalation of nalkylamines into misfit layer sulfides. J. Mater. Chem. 6, 861-866 (1996).

21. Lin, S. et al. Accessing valley degree of freedom in bulk Tin(II) sulfide at room temperature. Nat. Commun. 9, 1455 (2018).

22. Wiegers, G. A., Meetsma, A., Boer, J. L. D., Smaalen, S. V. \& Haange, R. J. X-ray crystal structure determination of the triclinic misfit layer compound (SnS) 1.20TiS2. J. Phys. Condens. Matter 3, 2603-2612 (1991).

23. Ettema, A. R. H. F., Wiegers, G. A., Haas, C. \& Turner, T. S. Resonant and core level photoemission spectroscopy of the misfit layer compound (SnS)1.20TiS2. Phys. Scr. T41, 265-268 (1992).

24. Raadik, T., Grossberg, M., Raudoja, J., Traksmaa, R. \& Krustok, J. Temperaturedependent photoreflectance of SnS crystals. J. Phys. Chem. Solids 74, 1683-1685 (2013).

25. Hawkins, C. G. \& Whittaker-Brooks, L. Vertically oriented TiS2-x nanobelt arrays as binder- and carbon-free intercalation electrodes for Li- and Na-based energy storage devices. J. Mater. Chem. A 6, 21949-21960 (2018).

26. Barriga, C., Lavela, P., Morales, J. \& Tirado, J. L. Chemical and electrochemical intercalation of lithium into SnTiS3 and BiTi2S5 misfit layer compounds. Solid State lonics 63-65, 450-456 (1993).

27. Park, T. H. et al. Shape-adaptable 2D titanium carbide (MXene) heater. ACS Nano 13, 6835-6844 (2019). 
28. Velický, M. et al. Exfoliation of natural van der Waals heterostructures to a single unit cell thickness. Nat. Commun. 8, 14410 (2017).

29. Deb, A. K. \& Kumar, V. Bandgap engineering in semiconducting one to few layers of SnS and SnSe. Phys. Status Solidi (B) 254, 1600379 (2017).

30. Alfakes, B. et al. Optoelectronic tunability of Hf-doped $\mathrm{ZnO}$ for photovoltaic applications. J. Phys. Chem. C. 123, 15258-15266 (2019).

31. Tamalampudi, S. R. et al. Rapid discrimination of chemically distinctive surface terminations in 2D material based heterostructures by direct van der Waals identification. Rev. Sci. Instrum. 91, 023907 (2020).

32. Amadei, C. A., Santos, S., Pehkonen, S. O., Verdaguer, A. \& Chiesa, M. Minimal invasiveness and spectroscopy-like footprints for the characterization of heterogeneous nanoscale wetting in ambient conditions. J. Phys. Chem. C 117, 20819-20825 (2013).

33. Santos, S. et al. A method to provide rapid in situ determination of tip radius in dynamic atomic force microscopy. Rev. Sci. Instrum. 83, 043707 (2012).

34. Plummer, A., Tang, T.-C., Lai, C.-Y. \& Chiesa, M. Nanoscale hydrophilicity studies of Gulf Parrotfish (Scarus persicus) scales. ACS Appl. Mater. Interfaces 6, 16320-16326 (2014).

35. Sader, J. E. \& Jarvis, S. P. Accurate formulas for interaction force and energy in frequency modulation force spectroscopy. Appl. Phys. Lett. 84, 1801-1803 (2004).

36. Sader, J. E. et al. Quantitative force measurements using frequency modulation atomic force microscopy?theoretical foundations. Nanotechnology 16, S94-S101 (2005).

37. Rajput, N. S. et al. Electron beam induced rapid crystallization of water splitting nanostructures. MRS Adv. 1, 825-830 (2015).

\section{ACKNOWLEDGEMENTS}

This work was funded under the Cooperative Agreement between the Khalifa University of Science and Technology, Masdar campus, Abu Dhabi, UAE, and the Massachusetts Institute of Technology (MIT), Cambridge, MA, USA, Reference Number FR2017-000001. M.C. acknowledges the support of the Arctic Center for Sustainable Energy (ARC), UiT Arctic University of Norway through grant no. 310059.

\section{AUTHOR CONTRIBUTIONS}

S.R.T. designed and conceived all the experiments. J.-Y.L. performed the DFT simulations. N.R. and S.P. performed TEM analysis. B.A. performed XRD and EDAX measurements. C.-Y.L. carried out AFM measurements. R.S. independently synthesized the bulk SnTiS 3 crystals. S.R.T., J.-Y.L., I.A., and M.C. analyzed and interpreted the results. S.R.T., J.-Y.L., H.A., B.A., and M.C. wrote the paper with input from all authors. All authors discussed the results.

\section{COMPETING INTERESTS}

The authors declare no competing interests.

\section{ADDITIONAL INFORMATION}

Supplementary information is available for this paper at https://doi.org/10.1038/ s41699-020-0158-7.

Correspondence and requests for materials should be addressed to J.-Y.L. or M.C.

Reprints and permission information is available at http://www.nature.com/ reprints

Publisher's note Springer Nature remains neutral with regard to jurisdictional claims in published maps and institutional affiliations.

(i) Open Access This article is licensed under a Creative Commons Attribution 4.0 International License, which permits use, sharing, adaptation, distribution and reproduction in any medium or format, as long as you give appropriate credit to the original author(s) and the source, provide a link to the Creative Commons license, and indicate if changes were made. The images or other third party material in this article are included in the article's Creative Commons license, unless indicated otherwise in a credit line to the material. If material is not included in the article's Creative Commons license and your intended use is not permitted by statutory regulation or exceeds the permitted use, you will need to obtain permission directly from the copyright holder. To view a copy of this license, visit http://creativecommons. org/licenses/by/4.0/.

(c) The Author(s) 2020 